\title{
ONE APPROACH TO THE SYNTHESIS, DESIGN AND MANUFACTURE OF HYPERBOLOID GEAR SETS WITH FACE MATING GEARS. PART 1: BASIC THEORETICAL AND CAD EXPERIENCE*
}

\author{
Valentin Abadjiev, Emilia Abadjieva \\ Institute of Mechanics, Bulgarian Academy of Sciences, \\ Acad. G. Bonchev St., Bl. 4, Sofia 1113, Bulgaria, \\ Graduate School of Engineering and Resource Science, \\ Faculty of Engineering and Resource Science, \\ Akita University, Tegatagakuen - machi 1-1, Akita, Japan \\ e-mails: abadjiev@imbm.bas.bg, abadjieva@gipc.akita-u.ac.jp
}

[Received 15 March 2016. Accepted 20 June 2016]

\begin{abstract}
Hyperboloid gear drives with face mating gears are used to transform rotations between shafts with non-parallel and non-intersecting axes. A special case of these transmissions are Spiroid ${ }^{1}$ and Helicon gear drives. The classical gear drives of this type are the Archimedean ones. The objective of this study are hyperboloid gear drives with face meshing, when the pinion possesses threads of conic convolute, Archimedean and involute types, or the pinion has threads of cylindrical convolute, Archimedean and involute types. For simplicity, all three types transmissions with face mating gears and a conic pinion are titled Spiroid and all three types transmissions with face mating gears and a cylindrical pinion are titled Helicon.

Principles of the mathematical modelling of tooth contact synthesis are discussed in this study. The presented research shows that the synthesis is realized by application of two mathematical models: pitch contact point and mesh region models. Two approaches for synthesis of the gear drives in accordance with Olivier's principles are illustrated. The algorithms and computer programs for optimization synthesis and design of the studied hyperboloid gear drives are presented.
\end{abstract}

\footnotetext{
${ }^{*}$ Corresponding author e-mail: abadjieva@gipc.akita-u.ac.jp.

${ }^{1}$ Spiroid, Helicon and Planoid are registered trade mark of Illinois Tool Works, Chicago, Illinois.
} 
KEY WORDs: Mathematical modelling, spatial gear drives, tooth contact synthesis, pitch contact point, mesh region, Computer Aided Design (CAD) program.

\section{Introduction}

The spatial transformation of rotations with constant angular velocities between fixed crossed axes is realized by three-links gear mechanisms, known as hyperboloid gears. This name is related to the form of their axoides, which are two mating hyperboloids of revolution, whose geometric axes coincide with the rotation axes. The contact line of the hyperboloids is the axis of screw motion of the rotation links. The dimensions and the geometry of the hyperboloids, which are determined in a unique way, depend on the parameters defining the dimensions and the structure of the gear set, as well as on the gear ratio, that characterize the type of rotation transformation.

Skew-axes gear pairs, transforming rotations by means of conjugate tooth surfaces are widely used among known motion transformers. They could realize the exact motion law (i.e. the gear ratio), they are designed to.

The successful use of hyperboloid gears with new kinematic and strength characteristics in industry makes slow progress because:

- common principles of transformation of rotations between skewed axes are not well examined;

- approaches to the mathematical modelling of synthesis of these types of mechanisms are not defined.

Spiroid gear pairs, which are the objectives of this study, belong to one of the most progressive and comparatively less applied in the engineering practice type of skew-axes gears. For the first time, we knew about this type of transmissions by F. Bohle [1], O. Saari [2], W. Nelson [3-5], D. Dudley [6, 7], etc. during the period 1955 - 1962. First patents are introduced in USA by Illinois Tool Works, Chicago at this time. This firm has constructed the rows of power classes and exactness tolerances classes of Spiroid gears and has organized the manufacture of Spiroid gear trains with a general function.

About 1962, Spiroid gearing excited the scientific interest of some researchers as Russian scientists A. Georgiev and V. Goldfarb [8-10], who have devoted a lot of papers as well as their Ph.D. thesis at this theme. D. Schwagerl [11], V. Bolos [12], D. Su, Y. Song and C. R. Gentle [13] have worked in this field, too.

After 1980, the first author of this paper has paid special attention to Spiroid gearing [14-23]. The theoretic researches on the geometry and kinematics of these transmissions, generalizing in his Ph.D. and D.Sc. thesis [14, 
23], are the basis of the mathematical modelling for their synthesis and design.

Among progressive combinations of spatial gears (see Fig. 1), Spiroid and Helicon gears occupy one essential place. The placement of the mesh region, geometry and technology of elaboration of these hyperboloid gear drives is the reason why they are treated as a hybrid between hypoid gear sets and worm ones [23]. For this reason, Spiroid and Helicon gear drives obtain constructive, technological and exploitation characteristics, that make them suitable for application as power and kinematic gear mechanisms, which ensure the smooth and the noiseless rotations transformation and with a possibility to realize gearing without a backlash between mating tooth surfaces.
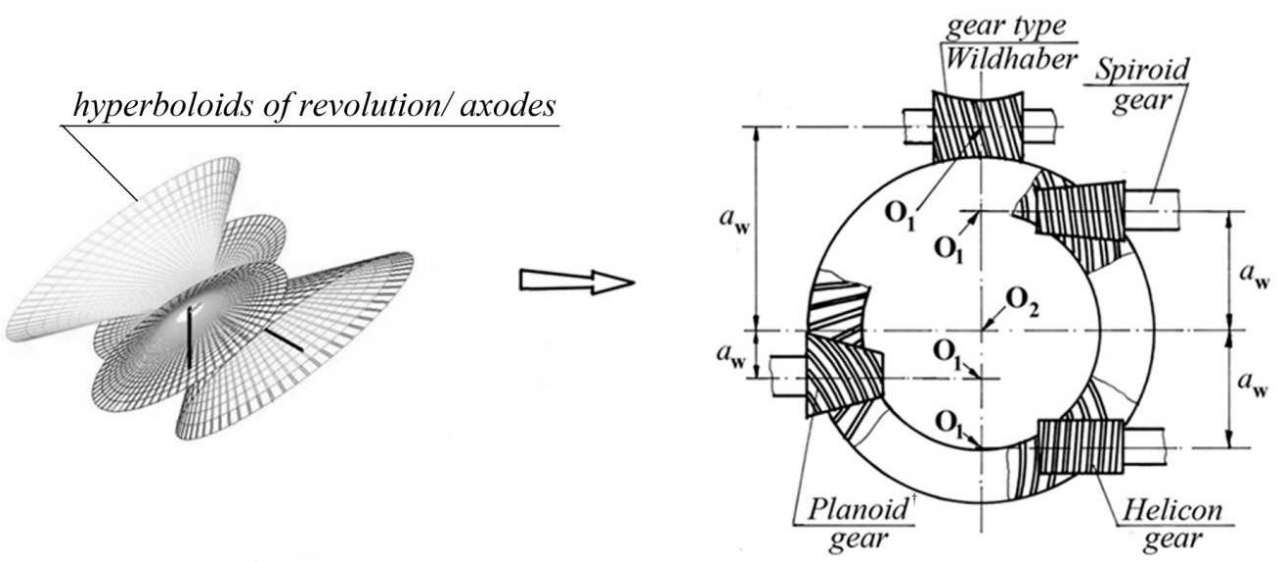

Fig. 1. Family of skew-axes gears

\section{Synthesis of skew axes gears of Spiroid and Helicon types}

Research into the spatial gearing, treated here, states that gear synthesis can be realized by applying two mathematical models [20,23], which will be discussed bellow.

\subsection{Synthesis using a pitch contact point}

The mathematical model for synthesis upon a pitch contact point is based on the assumption, that the necessary quality characteristics (that define concrete exploitation and technological requirements to the active tooth surfaces) are guaranteed only at one concrete point $P$ of the active tooth surfaces $\Sigma_{1}$ and $\Sigma_{2}$, and in its close vicinity. According to this model, the common contact point $P$ of the conjugate tooth surfaces $\Sigma_{1}$ and $\Sigma_{2}$ is the common point of both circles $H_{i}^{c}(i=1,2)$, that are called a pair of pitch circles $\left(H_{1}^{c}: H_{2}^{c}\right)$. 
The point $P$ is called a pitch contact point, the plane $T_{m}$, including the tangents to $H_{i}^{c}(i=1,2)$ at the point $P-a$ pitch plane and $m-m$ is the pitch normal to $T_{m}$ at the point $P$. The pair of rotation surfaces, that include the corresponding pairs of pitch circles and whose common normal at the point $P$ is the straight-line $m-m$, are an analogue of $H_{i}^{c}(i=1,2)$ and are called pitch surfaces $H_{i}^{s}(i=1,2)$. The pair of circles $\left(H_{1}^{c}: H_{2}^{c}\right)$ is directly related to the evaluation of the pitch and of the tooth module of the designed gear set. The pitch circles and the surfaces parameters define the dimensions of the reference surfaces, i.e. the blank proportions of the gears depend on them. These parameters are used, when the mountings dimensions of the synthesized gear-set are calculated.

Thus, the mathematical model for synthesis, based on a pitch contact point, ensures the solution of the two basic problems:

- synthesis of the pitch configurations;

- synthesis of the active tooth surfaces.

\subsubsection{Synthesis of the Pitch Configurations}

Pitch configurations: essence and definition. The present paper treats the kinematical theory of the spatial rotations transformation in the context of defining the kinematic-geometric essence of the basic elements of the mathematical models for synthesis of hyperboloid gears and their role in solving the gears synthesis. These elements have been called pitch configurations (pitch circles and pitch surfaces) and deal with one actual but still disputable (as content and terminology) part of the meshing theory. From the performed analysis of the publications $[20,23]$ and the comments above, we can conclude that:

- If the law of transformation of rotations $i_{12}=\omega_{1} / \omega_{2}=$ const. $\left(\bar{\omega}_{i}-\right.$ angular velocity of the rotating link $i$ ) between fixed and crossed axes $1-1$ and $2-2$ (the shortest distance between them is $a_{w}=$ const. and the angle between them is $\delta=\angle\left(\bar{\omega}_{1}, \bar{\omega}_{2}\right)=$ const. $)$ is given, and if the position of a point (treated as a point of contact of conjugate tooth surfaces $\Sigma_{1}$ and $\Sigma_{2}$ ) in the fixed space is known, then the diameters and the mutual position of the circles $H_{i}^{c}(i=1,2)$ are completely and uniquely determined. The circumferential velocity vectors $\bar{V}_{i}(i=1,2)$ of the common point $P$, and the relative velocity vector $\bar{V}_{12}$ at the same point, (i.e. the plane $T_{m}$, where the coplanar vectors $\bar{V}_{i}$ $(i=1,2)$ and $\bar{V}_{12}$ lie), as well as the normal $m-m$ to $T_{m}$ at the point $P$, are determined in a unique way, too.

- It is sufficient to know the mutual position of the crossed axes of rotation $1-1$ and $2-2$, and the position of the point $P$ (as a common point of 
the tooth surfaces $\Sigma_{1}$ and $\left.\Sigma_{2}\right)$ in the fixed space, in order the circles $(i=1,2)$ to be completely and uniquely determined (as diameters and mutual position). The plane $T_{m}$, formed by the tangents to the circles $H_{i}^{c}(i=1,2)$ at the point $P$, as the normal $m-m$ to $T_{m}$ at the point $P$, are uniquely determined, too.

In the most common case, the defined pitch configurations do not coincide with elements of the axodes of this class gear mechanisms, the axodes being hyperboloids of revolution.

Mathematical model for synthesis of pitch configurations. Let two crossed axes $1-1$ and $2-2$, representing the axes of rotations of the movable links of a three-link tooth mechanism, be given in the fixed space. Their mutual position is defined by the angle $\delta=$ const. (the angle between the angular velocity vectors $\bar{\omega}_{1}$ and $\bar{\omega}_{2}$ of the movable links $\left.(i=1,2)\right)$ and the shortest distance $a_{w}=$ const. The concrete study is performed when $\delta \in(0, \pi)$. Each pitch circle $H_{i}^{c}$ lies in a plane perpendicular to the axis of rotation $i-i$ of the movable link $i$ and has a radius, equal to the distance from the point $P$ to the axis $i-i$. The pitch plane $T_{m}$ is determined by the tangents to the circles $H_{1}^{c}$ and $H_{2}^{c}$ at the pitch contact point $P$. Beside, the pitch normal $m-m$ to $T_{m}$ is determined at $P$. The study performs by means of the notations and the coordinate frames $S_{1}\left(O_{1}, x_{1}, y_{1}, z_{1}\right)$ and $S_{2}\left(O_{2}, x_{2}, y_{2}, z_{2}\right)$, introduced in Fig. 2.

The dimensions and the mutual position of $H_{1}^{c}$ and $H_{2}^{c}$ are completely determined by the cylindrical coordinates $a_{i}, r_{i}, \theta_{i}(i=1,2)$ of the contact point $P$ in the systems $S_{i}(i=1,2)$ and by the angles $\delta_{i}(i=1,2)$, between the planes of $H_{i}^{c}(i=1,2)$ and the normal $m-m$. The pitch surfaces $H_{i}^{s}(i=1,2)-$ analogues of the pitch circles are illustrated in Fig. 2. The points of intersection $C_{i}^{\prime}(i=1,2)$ of the axes of rotation $i-i(i=1,2)$ and the plane $T_{m}$ are the tips of the pitch cones $H_{i}^{s}(i=1,2)$. Figure 2 is oriented to the synthesis of pitch configurations, whose common position in the fixed space ensures the possibility to design a big diversity of hyperboloid gears [23 - 25]. There, geometric pitch configurations with normal orientation in the fixed space are shown. These pitch configurations are typical for the traditional constructive types of spatial gears (hypoid ones, Spiroid and Helicon ones, etc.). They are characterized by: $\delta=\frac{\pi}{2}$ and $\delta \neq \frac{\pi}{2} ; z_{2, C_{2}}>0 ; \angle\left(\overline{C_{1} C_{1}^{\prime}}, \overline{O_{1} z_{1}}\right)=0^{\circ} ; \angle\left(\overline{C_{2} C_{2}^{\prime}}, \overline{O_{2} z_{2}}\right)=180^{\circ}$.

We represent the radius-vector $\overline{O_{1} P}$ and the unit vector $\bar{m}$ of the normal $m-m$ by $a_{1}, r_{1}, \theta_{1}$ and $a_{2}, r_{2}, \theta_{2}$ and using $a_{w}$ and $\delta$. Thus, we get the following set of equations: 


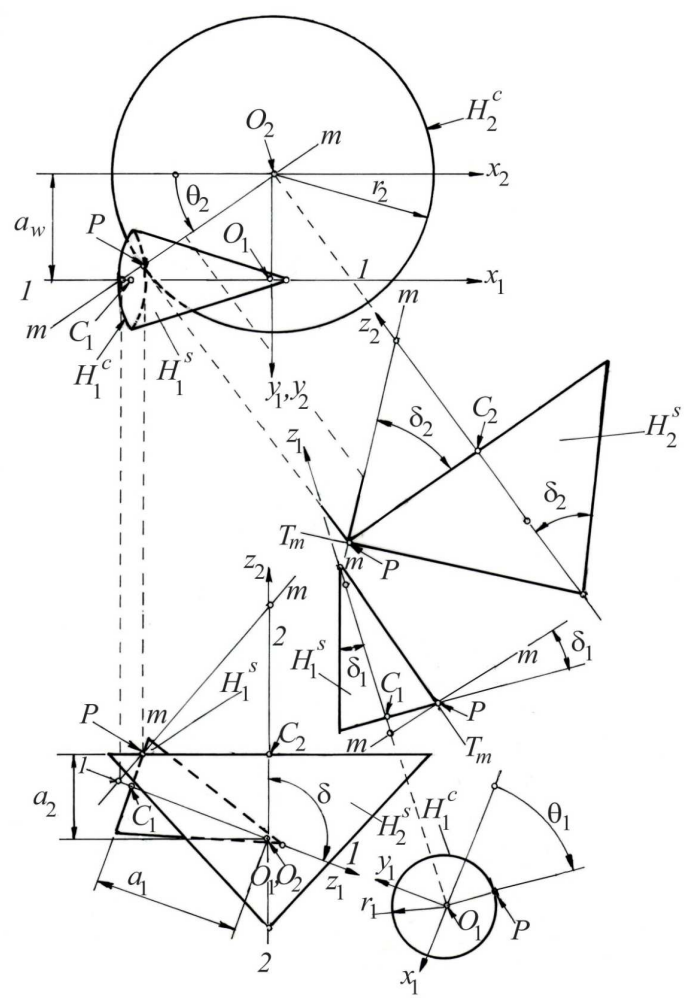

Fig. 2. Externally contacting pitch circles $H_{i}^{c}(i=1,2)$ and pitch surfaces $H_{i}^{s}$ $(i=1,2)$ with a normal orientation, corresponding to hyperboloid gears with external meshing

$$
\begin{aligned}
& r_{1} \cos \theta_{1}=r_{2} \cos \theta_{2} \cos \delta+a_{2} \sin \delta \\
& r_{1} \sin \theta_{1}=a_{w}-r_{2} \sin \theta_{2}, \\
& a_{1}=r_{2} \cos \theta_{2} \sin \delta-a_{2} \cos \delta \\
& \cos \delta_{1} \sin \theta_{1}=\cos \delta_{2} \sin \theta_{2}, \\
& \sin \delta_{1}=\sin \delta_{2} \cos \delta+\cos \delta_{2} \cos \theta_{2} \sin \delta, \\
& \cos \delta_{1} \cos \theta_{1}=\sin \delta_{2} \sin \delta-\cos \delta_{2} \cos \theta_{2} \cos \delta
\end{aligned}
$$

Later, the study of the system (1) will be performed taking into account the following geometric conditions $\theta_{1} \in\left[0, \frac{\pi}{2}\right], \theta_{2} \in\left(0, \frac{\pi}{2}\right], \delta_{1} \in\left[0, \frac{\pi}{2}\right)$, $\delta_{2} \in\left[0, \frac{\pi}{2}\right], a_{w}>0, \delta_{1}, a_{i} \geq 0,(i=1,2)$. Each of the last three equations in (1) is a consequence of the other two, i. e. (2) is a set of 5 independent 
equations with 10 unknowns: $\delta, a_{w}, \delta_{1}, r_{1}, a_{1}, \theta_{1}, \delta_{2}, r_{2}, a_{2}, \theta_{2}$. Therefore, each solution of (1) is a function of 5 of them (we will consider them as free ones). We suppose that the independent (free) parameters are $\delta, a_{w}, \delta_{1}, r_{1}$ and $a_{1}$. We will look for analytical relations, that they have to be fulfilled, so that the set (1) to have a solution.

Synthesis of orthogonal contacting pitch configurations $-\delta=\frac{\pi}{2}$. Let us pay attention not only to the cases oriented to the synthesis of Spiroid and Helicon gears, but also to such essential for the practice cases of orthogonal hyperboloid gears.

(a). $\delta_{1}=0$ and $a_{1} \neq 0$.

This case refers to the orthogonal hyperboloid gears with external meshing, when the coaxial surfaces of the movable link $i=1$ - reference, root and tip ones are of cylindrical form. System (1) has the following unique solution:

$$
\begin{aligned}
\theta_{1} & =0, \quad \tan \theta_{2}=\frac{a_{w}}{a_{1}}, \quad a_{2}=r_{1}, \\
r_{2} & =\frac{a_{w}}{\sin \theta_{2}}, \quad \delta_{2}=\frac{\pi}{2},
\end{aligned}
$$

in an arbitrary choice of $a_{w}, r_{1}$ and $a_{1}$. The parameters in (2) define the dimensions and the position of the pitch circles and surfaces, corresponding to spatial high reduction gears of type Helicon [6, 7]. This is the borderline case that separates externally and internally tangent geometric pitch configurations (Fig. 3).
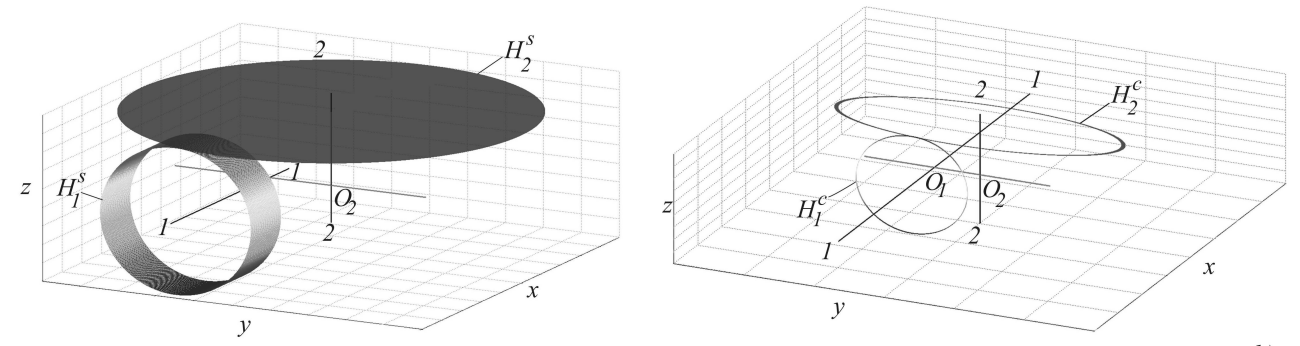

a)

b)

Fig. 3. Pitch configurations for gears of type Helicon: a) pitch surfaces $H_{i}^{s}(i=1,2)$; b) pitch circles $H_{i}^{c}(i=1,2) ; a_{w}=3.25 \mathrm{~mm} ; \delta=90^{\circ} ; \delta_{1}=0^{\circ} ; r_{1}=4 \mathrm{~mm} ; a_{1}=9.5$ $\mathrm{mm} ; \delta_{2}=90^{\circ} ; a_{2}=4 \mathrm{~mm} ; r_{2}=10.041 \mathrm{~mm}$

(b). $\delta_{1}>0$ and $a_{1} \neq 0$

In this case, the condition for an existence of geometric pitch configurations is $a_{1} \geq\left(a_{w}-r_{1}\right) \tan \delta_{1}$, and the system solution is: 

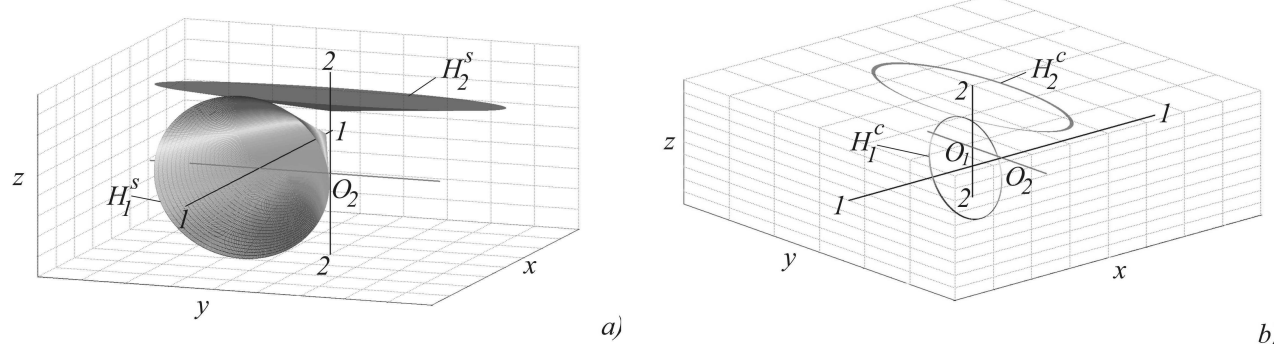

Fig. 4. Pitch configurations for orthogonal Spiroid gears: a) pitch surfaces $H_{i}^{s}$ $(i=1,2)$; b) pitch circles $H_{i}^{c}(i=1,2) ; a_{w}=3.25 \mathrm{~mm} ; \delta=90^{\circ} ; \delta_{1}=5^{\circ}$; $r_{1}=4 \mathrm{~mm} ; a_{1}=9.5 \mathrm{~mm} ; \delta_{2}=84^{\circ} ; a_{2}=3.9983 \mathrm{~mm} ; r_{2}=10.003 \mathrm{~mm}$

$$
\begin{aligned}
& \cot \theta_{2}=\frac{r_{1} \tan \delta_{1}+a_{1}}{a_{w}}, \quad \sin \theta_{1}=\frac{a_{w}}{r_{1}+a_{1} \cot \delta_{1}}, \\
& a_{2}=r_{1} \cos \theta_{1}, \quad \cos \delta_{2}=\frac{\sin \delta_{1}}{\cos \theta_{2}}, \\
& r_{2}=\frac{a_{w}-r_{1} \sin \theta_{1}}{\sin \theta_{2}} .
\end{aligned}
$$

The parameters of the pitch configurations of orthogonal hypoid gears and gears of type Spiroid $[6,7]$ are calculated by the relations given in (3). The pitch surfaces and the circles are visualized in Fig. 4.

\subsubsection{Synthesis of Tooth Surfaces}

The algorithm for the synthesis of the pitch configurations is based on the principles of the geometrical synthesis. Therefore, it cannot directly define the kinematic characteristics of the synthesized mechanisms, since the law of motion transformation is not presented in the algorithm.

The configurations start common rotation, when the law of motion transformation is included in the pitch configuration algorithm. Thus, a sliding velocity vector $\bar{V}_{12}$ can be defined at their common point. The value and the direction of $\bar{V}_{12}$ are important, since they determine the geometrical parameters of the conjugate tooth surfaces $\Sigma_{1}$ and $\Sigma_{2}$ of the pinion and gear.

The synthesis of the pinion thread surface $\Sigma_{1}$ is of essential interest, when the manufacture of the gear pair is based on Olivier's second principle. The synthesis of tooth surfaces upon a pitch contact point does not require knowing an analytic expression for the active flanks. The synthesis of the surface $\Sigma_{1}$ can be reduced to determine its basic geometric characteristics at the pitch point $P$. Thus, the synthesis algorithm comprises analytical relations, defining sufficient number of parameters of the active flanks $\Sigma_{1}$ by means of 
which the conditions for design of both the Spiroid pinion and the Spiroid hob are created. Here, it should especially be noted, that specific constructive characteristics, which are necessary for the design and the elaboration of the Spiroid pinion and the Spiroid hob with convolute, Archimedean or involute active tooth surfaces of the pinion threads, are determined by using of the calculated parameters in the pitch contact point. The Spiroid hob is used for the Spiroid gear $\Sigma_{2}$ tooth cutting.

Here, some of the analytic relations, included in algorithms and computer programs for Spiroid and Helicon gear synthesis, are presented. Expressions given below correspond to gears with right-hand pinion threads and left-hand gear teeth. The directions of the angular velocities vectors $\bar{\omega}_{1}$ and $\bar{\omega}_{2}$, shown in Fig. 5, correspond to the mentioned orientation of the tooth surfaces $\Sigma_{1}$ and $\Sigma_{2}$.

(a) Helix angle of the pinion thread flank. This angle gives the direction of the helix of the helical flank $\Sigma_{1}$ in the pitch point $P . \beta_{1}$ is the angle between the tangent $t-t$ to the helix $L_{1}$ in $P$ and the straight line $P O_{1}^{\prime}$, passing through $P$ and concluding angle $\delta_{1}$ with the axis $1-1$ (see Fig. 5):

$$
\beta_{1}=\arctan \frac{i_{12} r_{1}-r_{2} \cos \mu}{r_{2} \sin \mu}, \quad \mu=\arcsin \frac{\sin \theta_{2} \sin \delta}{\cos \delta_{1}} .
$$

(b) Axial helical parameter and axial module. The axial helical parameter is a basic geometrical parameter of the pinion. By using it, the pinion helix pitch can be determined. Its expression is [23]:

$$
p_{s}=\frac{r_{1} r_{2} \cos \delta_{1} \sin \mu}{i_{12} r_{1}-r_{2} \cos \mu} .
$$

Assuming $\delta=\pi / 2$ and $\delta_{1}=0$ in (4) and (5), analytical expressions for $\beta_{1}$ and $p_{s}$, corresponding to Helicon pinion, are obtained.

By using equation (5), it is easy to determine the axial pitch and axial module of the Spiroid pinion:

$$
P_{s}=2 \pi \frac{p_{s}}{z_{1}}, \quad m_{s}=2 \frac{p_{s}}{z_{1}} .
$$

Here, $z_{1}$ is number of Spiroid pinion threads $\left(z_{2}\right.$ is number of Spiroid gear teeth).

The axial pitch and the axial module are basic geometrical characteristics of the gear sets of the considered class.

(c) Pinion thread pressure angles. It is possible to obtain singular points in the mesh region, when meshing mutually enveloping surfaces $\Sigma_{1}$ and $\Sigma_{2}$. 


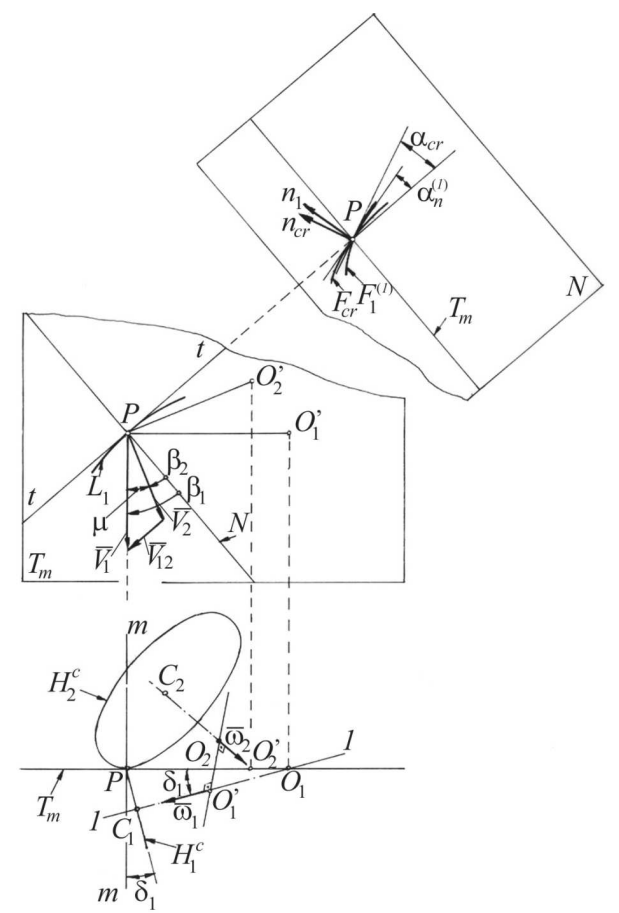

Fig. 5. Spiroid gear tooth geometry

Such points exist, if the relative velocities of the conjugate tooth surface contact points are zero [23]:

$$
\bar{V}_{r, i}=\overline{0}
$$

Depending on the normal vector $\bar{n}_{i}$ to the meshed surfaces at their common point, two types of singular points exist:

- singular points of first order, called ordinary nodes if $\bar{n}_{i} \neq \overline{0}$, and consequently $\dot{\bar{n}}_{r, i}=\overline{0}$, where $\dot{\bar{n}}_{r, i}$ is the relative velocity vector of the top of the normal vector in its relative motion;

- singular points of second order, called points of undercutting, when $\bar{n}_{i}$ does not exist.

Singular points of first order should be eliminated from the mesh region, since they increase specific friction, reduce lubrication and heat transfer, and as a result they decrease loading capacity of the gear pair (because of the increased relative sliding).

The aim is to eliminate the ordinary nodes in the pitch point vicinity, in the cases of Spiroid gear synthesis, using a pitch contact point $P$. To carry 
out this task, a limiting direction of the normal vector $\bar{n}_{1}$, with respect to the tooth surface $\Sigma_{1}$ at point $P$, is sought. Here, it is considered that a point of $\Sigma_{1}$ is an ordinary node. The analytic expression of the angle between this vector and the plane $T_{m}$ (see Fig. 5) $[17,23]$ is given below:

$$
\alpha_{c r}=\arctan \left\{\begin{array}{l}
\sin \beta_{1}\left[r_{1} \cos \delta_{1}+a_{w} \cot \delta\left(\cot \beta_{1}+\right.\right. \\
\left.\left.+\tan \theta_{1} \sin \delta_{1}\right)+a_{1}\left(\cot \beta_{1} \tan \theta_{1}-\sin \delta_{1}\right)\right] \\
r_{1} \sin \delta_{1}+a_{1} \cos \beta_{1}-a_{w} \cot \delta \tan \theta_{1} \cos \delta_{1}
\end{array}\right\} .
$$

The point $P$ will not be an ordinary node, if the normal vector to $\Sigma_{1}$ shifts, with respect to critical normal vector $\bar{n}_{c r}$, by the tooth standard profile angle $\alpha$. As a result, the pinion thread becomes non-symmetric in the normal section and the real profile angles in the normal section of the thread (i. e. the pressure angles) are of the form:

$$
\alpha_{n}=\alpha \mp\left|\alpha_{c r}\right|,
$$

The smaller value of $\alpha_{n}$ determines the orientation of the surfaces $\Sigma_{1}$. They belong to the pinion threads, when the pinion meshes with the Spiroid gear s corresponding surfaces $\Sigma_{2}$ and the angular velocity vectors are those, shown in Fig. 5.

(d) Special force angle. The special force angle $\alpha_{12}$ is the angle between the vector of the force transferred from $\Sigma_{1}$ to $\Sigma_{2}$ and the circumferential velocity vector of the pitch contact point $P$, being a point of $\Sigma_{2}$ :

$$
\cos \alpha_{12}=\cos \beta_{2} \cos \alpha_{n},
$$

where $\beta_{2}=\beta_{1}-\mu$.

The value of the special force angle has a critical effect on the conditions of force transmission between the conjugated surfaces $\Sigma_{1}$ and $\Sigma_{2}$. It also affects the synthesized mechanical transmission efficiency.

The basic geometric-kinematic characteristics, which are part of the analytical algorithm for optimization synthesis, are presented.

\subsubsection{Computer aided design (CAD) program}

This program consists of solving the following problems:

- synthesis of the pitch circles;

- synthesis of the tooth surfaces and of the cutting tool (Spiroid hob);

- verifying the quality criteria to be fulfilled. 
The question of the choice of the pitch contact point place is essential, when designing Spiroid gear pairs. It directly corresponds to the approach to mathematical modelling, using a pitch contact point. It has been already mentioned, that this point is a common point both of the pitch circles and of the conjugated tooth surfaces. That's why its place has influence on the gears proportions and on the gear pair quality (this is expressed by the geometric, kinematic and strength characteristics of the gear pair).

The pitch circles are two tangent circles (two circles with only one contact point). The center of one of them lies on the Spiroid pinion axis $1-1$ and its plane is perpendicular to $1-1$, while the center of the other belongs to the Spiroid gear axis $2-2$ and its plane is perpendicular to $2-2$. Mutual position of the skewed axes $1-1$ and $2-2$ is uniquely determined by the angle $\delta$ between the skewed axes and by the offset (center distance) $a_{w}$.

The position of each circle in respect with its axis and with the offset line is described by four parameters. Two vector equations (equivalent to 5 independent scalar equations) define the condition two circles to have one common point. Therefore, the position of two pitch circles is not defined in a unique way but is a function of 5 independent parameters. It rests to decide which they are and how to choose the intervals of their variations. We have decided these five free parameters to be: angle $\delta$, offset $a_{w}$, Spiroid pinion taper angle $\delta_{1}$, Spiroid pinion pitch circle radius $r_{1}$, distance $a_{2}$ between Spiroid gear pitch circle plane and the offset line.

Thus, a lot of couples of pitch circles are obtained, when varying the free parameters within given intervals. We look for such couples so that their parameters to fulfil a definite quality of gear pair in the pitch point vicinity. These criteria will be discussed below.

It should be mentioned, that the program gives the possibility to determine the type of helicoid will represent the surface of Spiroid threads: convolute (in the most common case), Archimedean or involute. The calculation of the necessary and sufficient geometric and technologic parameters for designing the gear pair and the tools for cutting is performed for the desired kind of helicoid.

Quality indices of the synthesized gear set are of essential significance in the tooth mechanism design process. In the case of the Spiroid gears design, the quality criteria that should be fulfilled in the pitch point vicinity are related to the technology, the gears geometric parameters, strength and kinematic characteristics. We will briefly describe some of them [23].

The basic criterion is in direct relation with the Spiroid gear pair manufacture technology. The decrease in cutting tools nomenclature involves the necessity to design the Spiroid pinion and the Spiroid hob with a standard 
module (pinion and hob parameters are functions of this module). Thus, it is natural to require that the calculated in the pitch contact point module to coincide (with a given exactness) with some of the standard modules, that form a set in the program system.

Another important criterion refers to the singular points of first order, that are called ordinary nodes. They are the common points of two infinitesimally close contact lines, i. e. such a point has an annular relative velocity vector, but the normal vector to the tooth surface in this point exists. The existence of such points in the pitch point vicinity involves decreasing of the loading capacity, of the efficiency and of the Spiroid gear pair durability. The elimination of the ordinary nodes is realized by inclining the normal vector to the tooth surface by a definite critical angle. Thus, the pressure angles of the tooth profile are not equal, i. e. the tooth is not symmetric in a normal section. Analytical relations between the chosen free parameters that guarantee non-existence of ordinary nodes in the pitch point vicinity are obtained and are included in the program system.

Control of the angle of normal force transmission. This angle depends on the Spiroid gear helix angle and on the low-side pressure angle. Smaller values of this angle guarantee better transmission of the forces in the pitch point vicinity and larger efficiency values.

Criterion for sliding velocity value. Maximum values, that limit this velocity depend on the materials, the Spiroid pinion and the Spiroid gear is produced of, i. e. different kinds of bronze or of steel.

Requirements, treating the efficiency, the conditions of cutting, etc. can be taken into account, also.

Some of the free parameters could be fixed (if the user wants to), because of the concrete requirements, such as limits for overall dimensions, manufacture of Spiroid gear sets, etc. Then, the number of the free parameters decrease, but this does not limit the possibility to look for and to obtain an optimal geometry of the tooth surfaces.

Input data of the computer program are: number of Spiroid pinion threads, number of Spiroid gear teeth, offset, standard pressure angle, kind of the Spiroid pinion, type of bearing mounting, frequency of revolution. Keys parameters with values of 1 or 0 , depending on whether a given criterion will be taken in consideration or not are introduced too. For each of the free parameters minimum and maximum values as well as the steps of variation should be chosen. The independent cycles in the computer program are equal to the free parameters.

You can see the scheme of the program in Fig. 6. The table with 


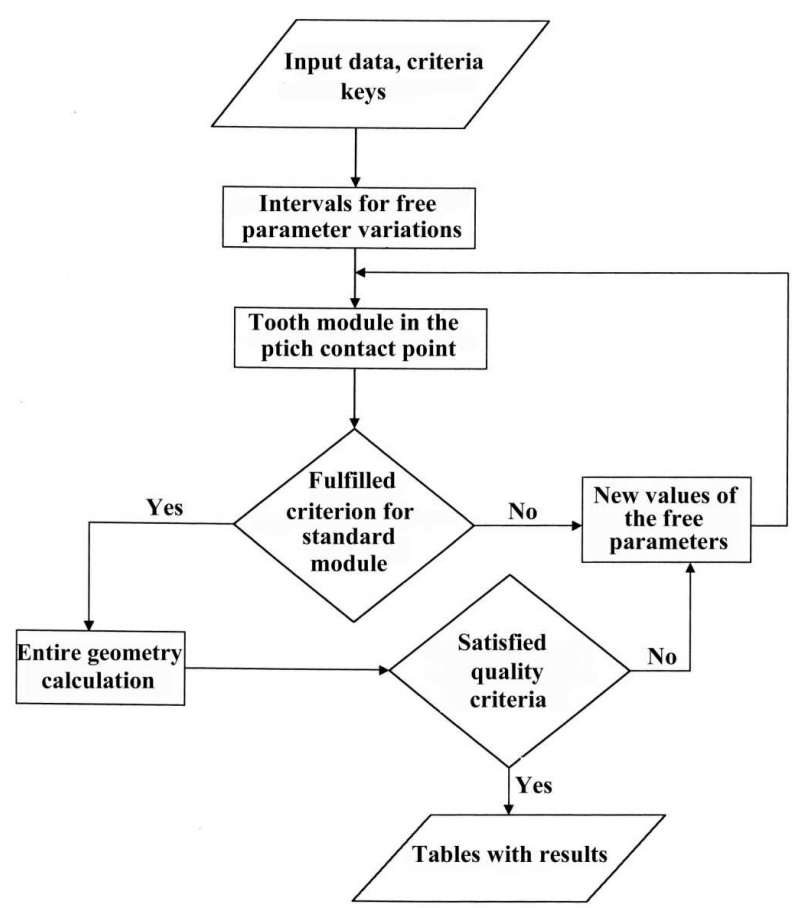

Fig. 6. Program scheme for a Spiroid gear pair optimizing synthesis

results consists of: the basic geometric parameters of the Spiroid pinion and of the Spiroid gear, the constructive parameters of the Spiroid hob, geometric and constructive parameters of the gear pair, parameters related to the gear pair quality, such as forces in the pitch contact point, distances between bearings and pitch circles planes, efficiency, etc. It should be mentioned, that distances may be calculated in two ways: as real dimensions or referred to the gear pitch circle radius. First, all forces and loads have relative values (i. e. they are referred to the gear peripheral force). If a torque on gear shaft or a torque on a pinion shaft is given, then this peripheral force has a concrete value and all forces and loads are calculated in $[\mathrm{N}]$. The sliding velocity is calculated, referred to the pinion angular velocity. If the numbers of the driving motor revolutions per minute are given, then the sliding velocity is obtained in $[\mathrm{m} / \mathrm{s}]$.

\subsection{Synthesis upon mesh region}

The presented here briefly algorithm and CAD program for synthesis upon a pitch contact point are the base of the applied by authors a 3D software technology for elaboration of the studied spatial gear drives. The approach to 
the synthesis upon a mesh region is the second important element of the above mentioned technology, that implements 3D printing. An essence of the modelling of the skew-axes gears of Spiroid and Helicon types by using the mesh region, is creating of the kinematic model of action tooth surfaces. The optimization process consists in determining the optimal parts of the mesh region, respectively the optimal parts of the active tooth surface of the synthesized pinion and gear. The synthesis upon a mesh region covers two main tasks: synthesis of the thread flanks of the Spiroid/Helicon pinion; synthesis of mesh region and tooth flanks of the Spiroid/Helicon gear. On their basis, the elimination of the singular points of first and second order (undercutting points) from the mesh region is performed.

After solving all the defined problems, which form the synthesis upon a mesh region, next step is $3 \mathrm{D}$ software generation of the tooth active surface of the gears. This is realized by 3D computer model, elaborated by 3D CAD software such as SolidWorks, Autodesk.

A basis of the mathematical modelling of the skew-axes gears of type Spiroid and Helicon, by using the mesh region, is the design of the kinematic model of the action surfaces. The optimization process consists in determining the optimal borders of the mesh region. A kinematic scheme of the Spiroid gear pair, related to the considered mathematical model is shown in Fig. 7.

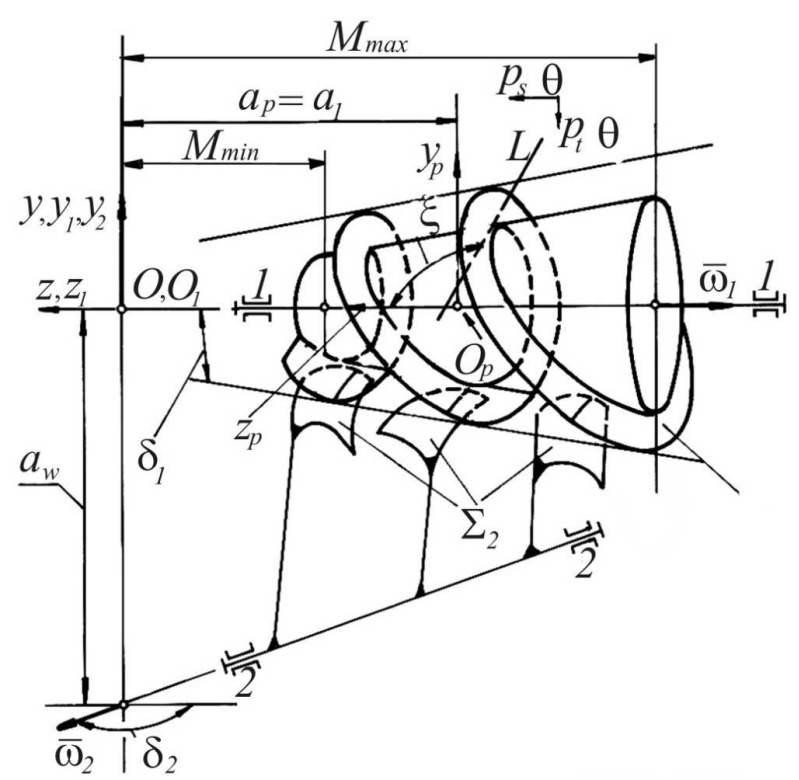

Fig. 7. Kinematic scheme of Spiroid gear pair 


\subsubsection{Synthesis of the thread flanks of the Spiroid pinion}

It has already been noted, that the technology of the considered gears manufacture is based on Olivier's second principle. The surface of action and the mesh region depend on the Spiroid pinion flanks $\Sigma_{1}$. According to [20, 23], $\Sigma_{1}$ are linear conic helicoids with constant axial parameter $p_{s}=$ const.

The synthesis of the Spiroid pinion with right-hand threads will be examined without restricting the generality of the problem. The generation of $\Sigma_{1}$ is shown in Fig. 6. In the most common case, $\Sigma_{1}$ is a convolute conic helicoids, which generatrix $L$ is a straight-line and performs a complex motion consisting of $[20,23]$ : a) helical motion along the axis $O_{p} z_{p}$ with parameter $p_{s}=$ const.; b) translation perpendicularly to the axis $O_{p} z_{p}$ with a parameter $p_{t}$ in the plane, which is a tangent to the cylinder with radius $r_{0}$. The generetrix $L$ does not cross the axis $O_{p} z_{p}$, which coincides with the geometric axis of the pinion. $L$ and $O_{p} z_{p}$ conclude an angle $0.5 \pi<\xi<\pi$. Then the equations of $\Sigma_{1}$ in the system $S_{p}\left(O_{p}, x_{p}, y_{p}, z_{p}\right)$ are:

$$
\begin{aligned}
& x_{p}=r_{0} \cos \theta \pm\left(u \sin \xi-p_{t} \theta\right) \sin \theta, \\
& y_{p}=r_{0} \sin \theta \mp\left(u \sin \xi-p_{t} \theta\right) \cos \theta, \\
& z_{p}=p_{s} \theta \pm u \cos \theta .
\end{aligned}
$$

Here, $\theta$ and $u$ are coordinates of helical surface $\Sigma_{1}$. The upper signs in (11) refer to the surface $\Sigma_{1}$, which rotates with angular velocity vector, $\bar{\omega}_{1}$ and the corresponding angular velocity vector of $\Sigma_{2}$ is $\bar{\omega}_{2}$ (see Figs 5 and 7 ). The lower signs in (11) are related to the meshed surfaces $\Sigma_{1}$ and $\Sigma_{2}$, whose angular velocity vectors of rotations are $-\bar{\omega}_{1}$ and $-\bar{\omega}_{2}$. Equations (11) describe an involute conical helical surface, if the following condition is satisfied:

$$
h=p_{s}+\left(r_{0} \pm p_{t}\right) \cot \xi=0 .
$$

Here, $h$ is a parameter of distribution [20, 23]. If $r_{0}=0$, then (11) are the equations of an Archimedean conical helicoid. The condition $p_{t}=0$ transforms equations (11) into a set of equations of cylindrical linear helical surfaces. Such surfaces are the active flanks of the pinion of type Helicon.

The equations of left-hand linear conic helical surfaces $\Sigma_{1}$ of a Spiroid pinion are:

$$
\begin{aligned}
& x_{p}=r_{0} \cos \theta \mp\left(u \sin \xi+p_{t} \theta\right) \sin \theta, \\
& y_{p}=r_{0} \sin \theta \pm\left(u \sin \xi+p_{t} \theta\right) \cos \theta, \\
& z_{p}=p_{s} \theta \pm u \cos \theta .
\end{aligned}
$$



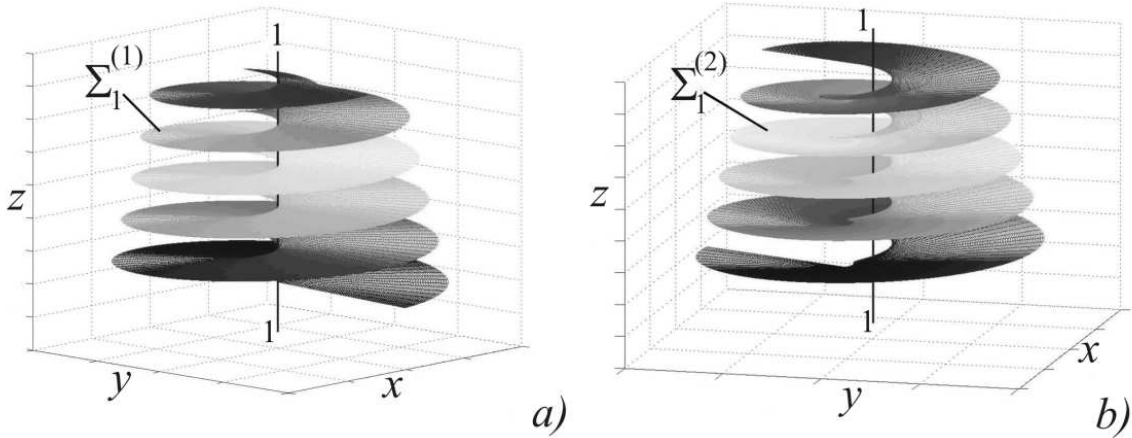

Fig. 8. Conic convolute helicoids: a) $\Sigma_{1}^{(1)} \Rightarrow \xi^{(1)}=98^{\circ}, r_{0}^{(1)}=0.08 \mathrm{~mm}$; $u^{(1)} \in[0,10], \vartheta^{(1)} \in[0,5 \pi]$; b) $\Sigma_{1}^{(2)} \Rightarrow \xi^{(2)}=120^{\circ}, r_{0}^{(2)}=0.94 \mathrm{~mm} ; u^{(2)} \in[0,10]$, $\vartheta^{(2)} \in[0,5 \pi]$
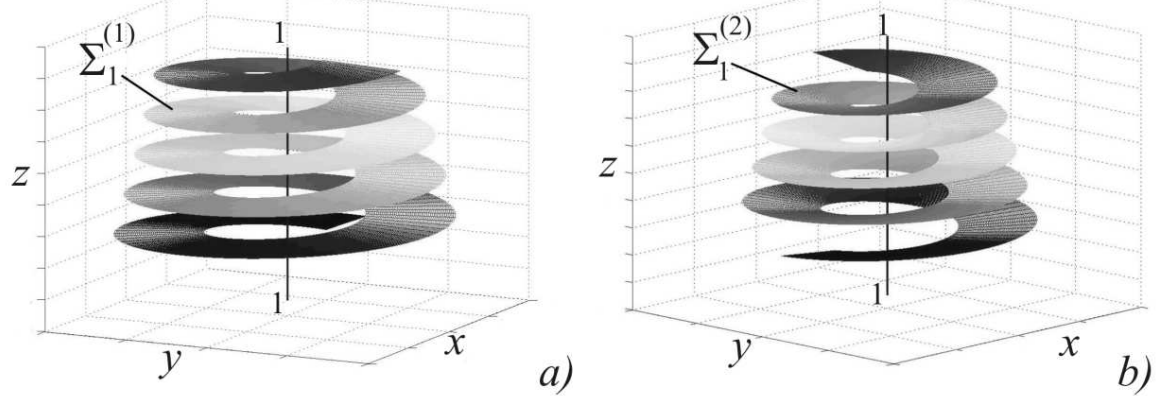

Fig. 9. Conic Archimedean helicoids: a) $\Sigma_{1}^{(1)} \Rightarrow \xi^{(1)}=98^{\circ}, u^{(1)} \in[5,10]$, $\vartheta^{(1)} \in[\pi / 2,10 \pi]$; b) $\Sigma_{1}^{(2)} \Rightarrow \xi^{(2)}=120^{\circ}, u^{(2)} \in[5,10], \vartheta^{(2)} \in[\pi / 2,10 \pi]$
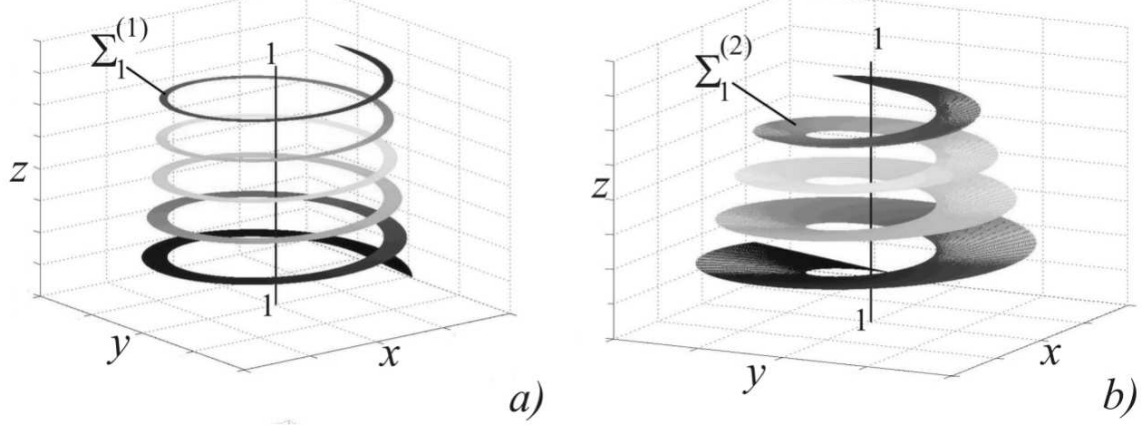

Fig. 10. Conic involute helicoids: a) $\Sigma_{1}^{(1)} \Rightarrow \xi^{(1)}=98^{\circ}, r_{0}^{(1)}=12.93 \mathrm{~mm}$, $u^{(1)} \in[0,10], \vartheta^{(1)} \in[0,10 \pi]$; b) $\Sigma_{1}^{(2)} \Rightarrow \xi^{(2)}=120^{\circ}, r_{0}^{(2)}=3,44 \mathrm{~mm}, u^{(2)} \in[0,10]$, $\vartheta^{(2)} \in[0,10 \pi]$ 
The following three figures (Figs 8-10) illustrate 3D graphics of conic linear helicoids, which are used as an active tooth surfaces of the Spiroid pinion's threads [26].

The upper index $(j)$ of $\Sigma^{(j)}, \xi^{(j)}, u^{(j)}$, for $j=1$ corresponds to the upper signs in the equations systems (11), and for $j=2$-lower signs.

\subsubsection{Synthesis of a mesh region}

A defined kinematic conjugation of the joint $\left(\Sigma_{1}: \Sigma_{2}\right)$ requires the geometrical elements, i. e. the tooth surfaces $\Sigma_{1}$ and $\Sigma_{2}$ are analytically described by functions, that are continuous and have continuous derivatives of first order. In addition, it is required that $\Sigma_{1}$ and $\Sigma_{2}$ have one conjugate point at least. The kinematic conjugation of the joint $\left(\Sigma_{1}: \Sigma_{2}\right)$ defines the motion transformation from each geometrical element $\Sigma_{1}$ to geometrical element $\Sigma_{2}$. In the concrete case of skew-axes gears with fixed axes and constant angular velocity vectors of the gears, the following specific restrictions, concerning the tooth surfaces $\Sigma_{1}$ and $\Sigma_{2}$ are imposed: representing the contact at one conjugate point $P$ at given moment, as a contact between two infinitesimally small areas of $\Sigma_{1}$ and $\Sigma_{2}$, which lie in a common tangent plane $T$. Then, it is evident, that these areas have a relative sliding, which is performed with the velocity vector:

$$
\bar{V}_{12}=\bar{V}_{1}-\bar{V}_{2} \text {. }
$$

lies in the plane $T$. Since the relative velocity vector $\bar{V}_{r, i}(i=1,2)$ of the conjugate point also lies in $T$, then normal components of the absolute velocity vectors are equal, i. e. $\bar{V}_{n, 1}=\bar{V}_{n, 2}$ and vice versa. This is true for every kinematically conjugated joint $\left(\Sigma_{1}: \Sigma_{2}\right)$. Therefore:

$$
\bar{n}_{i} \bar{V}_{12}=\bar{n}_{i}\left(\bar{V}_{1}-\bar{V}_{2}\right)=0 .
$$

Equation (14) is known as a basic equation of meshing [27, 28] and defines the condition of the existence of a common tangent plane $T$ in the conjugate contact point $P$ of the kinematically conjugate joint $\left(\Sigma_{1}: \Sigma_{2}\right)$ at a given moment. Thus, equation (14) is a geometrical and a kinematic relation, that defines the position of the conjugate contact points, respectively the contact lines on the tooth surfaces $\Sigma_{1}$ and $\Sigma_{2}$. If this is applied to the cases of Spiroid and Helicon gears, their mesh regions are as follows:

$$
\begin{aligned}
& x=r_{0} \cos \Phi \pm R_{0} \sin \xi \sin \Phi, \\
& y=r_{0} \sin \Phi \mp R_{0} \sin \xi \cos \Phi, \\
& z=p \theta \pm R_{0} \cos \xi-a_{p} \\
& A R_{0}^{2}+B R_{0}+C=0
\end{aligned}
$$


where: $\Phi=\varphi_{1}+\theta, R_{0}=u-p_{t} \theta / \sin \xi, p=p_{s} \pm p_{t} \cot \xi$,

$$
A= \pm i_{21} \sin \delta \cos \Phi
$$

$B=h\left[\left(1-i_{21} \cos \delta\right) \sin \xi-i_{21} \sin \delta \cos \xi \sin \Phi\right]+i_{21} \sin \delta\left(p \theta-a_{p}\right) \cos \xi \cos \Phi+$

$+i_{21}\left(a_{w} \cos \delta \cos \xi-r_{0} \sin \delta \sin \xi\right) \sin \Phi-\left(1-i_{21} \cos \delta\right) r_{0} \cos \xi-a_{w} i_{21} \sin \delta \sin \xi$

$$
C=\mp h\left[-a_{w} i_{21} \cos \delta \cos \Phi+i_{21} \sin \delta\left(p \theta-a_{p}\right) \sin \Phi\right] .
$$

Equations (15) describe the action surface, respectively the mesh region of a convolute Spiroid gear pair (i. e. $\Sigma_{1}$ is a convolute conical surface). For $\varphi_{1}=$ const., the analytical relation between $u$ and $\theta$ defines one contact line. If it is assumed in addition, that $r_{0}=0 ; h=0 ; p_{t}=0$ and $\delta=\pi / 2$, the surface of action, the mesh region of Archimedean Spiroid gear pair; involute Spiroid gear pair; and a gear pair of type Helicon are obtained, respectively.

\section{region}

\subsubsection{Test for points of undercutting existence in the mesh}

It is essential, to point out the singular points mentioned in Section 2.1, when synthesizing the examined spatial gears. Moreover, the dimensions of $\Sigma_{1}$ and $\Sigma_{2}$ are limited in the design process, so that the singularity in the mesh region is eliminated. Here, the singular points of second order (the points of undercutting) are discussed.

In the case of Spiroid and Helicon gears, the phenomenon "undercutting of $\Sigma_{2}$ " means that the fillet surface generated by the tip edge of the tool surface $\Sigma_{J}=\Sigma_{1}$ remove a part of the active flanks $\Sigma_{2}$. (The tip edge of $\Sigma_{J}$ is the line of intersection of $\Sigma_{J}$ with the addendum surface of the cutting part of the tool.) As a rule, the undercutting makes the tooth thinner [27].

Using equation (7), it is easy to obtain a vector equation, defining those points of the surface $\Sigma_{J}=\Sigma_{1}$, which correspond to the points of undercutting in the process of instrumental meshing:

$$
\bar{V}_{r 1\left(s_{p}\right)}+\bar{V}_{12\left(s_{p}\right)}=\overline{0} .
$$

Using equation (16), it is possible to find one line on the surface $\Sigma_{1}$ (considered as a tool surface), which limits this surface.

The last equation in (15) is an analytical expression of the basic meshing equation (14), and it can be written in the form:

$$
f\left(u, \theta, \varphi_{1}\right)=0 .
$$


Thus, the analytic conditions, defining the line of the undercutting points on the action surface, and on the mesh region, are:

$$
\begin{aligned}
& \bar{\rho}=x\left(u, \theta, \varphi_{1}\right) \bar{i}+y\left(u, \theta, \varphi_{1}\right) \bar{j}+z\left(u, \theta, \varphi_{1}\right) \bar{k} \\
& f\left(u, \theta, \varphi_{1}\right)=0, \\
& \frac{\partial f}{\partial u} \frac{d u}{d t}+\frac{\partial f}{\partial \theta} \frac{d \theta}{d t}+\frac{\partial f}{\partial \varphi_{1}} \frac{d \varphi_{1}}{d t}=0, \\
& \frac{V_{12, x}}{V_{r 1, x}}=\frac{V_{12, y}}{V_{r 1, y}}=\frac{V_{12, z}}{V_{r 1, z}}=-1
\end{aligned}
$$

All of equations (18) are written in the coordinate system $S(O, x, y, z)$ (see Fig. 7). In the case of the non-orthogonal Spiroid gear pair shown in Fig. 7 , the coordinates of the sliding velocity vector $\bar{V}_{12}$ and the equations of $\Sigma_{1}$ written in $S(O, x, y, z)$ are:

$$
\begin{aligned}
V_{12, x} & =-a_{w} i_{21} \cos \delta+\left(1-i_{21} \cos \delta\right) y, \\
V_{12, y} & =i_{21} \sin \delta z-\left(1-i_{21} \cos \delta\right) x, \\
V_{12, z} & =-i_{21} \sin \delta\left(a_{w}+y\right) \\
& \\
x & =r_{0} \cos \Phi \pm R_{0} \sin \xi \sin \Phi \\
y & =r_{0} \sin \Phi \mp R_{0} \sin \xi \cos \Phi, \\
z & =p \theta \pm R_{0} \cos \xi-a_{p} .
\end{aligned}
$$

\subsubsection{Elimination of the singular points of first order from} the mesh region

As already mentioned, one point belonging to the mesh region of a spatial gear pair is a singular point of first order, if the following conditions are satisfied:

$$
\begin{aligned}
& \bar{V}_{r, 1}=\frac{\partial \bar{\rho}_{1}}{\partial u} \frac{d u}{d t}+\frac{\partial \bar{\rho}_{1}}{\partial \theta} \frac{d \theta}{d t}=\overline{0}, \quad \varphi_{1}=\text { constant } \\
& \bar{n}_{1}=\frac{\partial \bar{\rho}_{1}}{\partial u} \times \frac{\partial \bar{\rho}_{1}}{\partial \theta} \neq \overline{0}
\end{aligned}
$$

Taking into account equations (21), as well as the third equation in (18), it is proved, that:

$$
\frac{\partial f}{\partial \varphi_{1}} \neq 0 .
$$

The value (22) is a condition for the non-existence of singular points of first order in the mesh region of the synthesized gear set. From (22), we 
can write three groups analytical conditions, connecting the geometric and the kinematic characteristics of Spiroid and Helicon gear sets. If we take them into account, conditions for elimination of the singularity of first order in the process of synthesis and design of the convolute, Archimedean and involute gears of the mentioned types are created. The critical normal vector $\bar{n}_{c r}$ to $\Sigma_{J}$, respectively to $\Sigma_{1}$ in an ordinary node satisfied equations [27]:

$$
\bar{n}_{c r} \cdot\left(\bar{V}_{1}-\bar{V}_{2}\right)=0, \quad \bar{n}_{c r} \cdot\left(\bar{\omega}_{1} \times \bar{V}_{1}-\bar{\omega}_{2} \times \bar{V}_{2}\right)=0 .
$$

Here, the first equation is the basic equation of meshing, when the contact point is an ordinary node. The second equation is a derivative of the first one with conditions $\omega_{1}=$ constant and $\omega_{2}=$ constant. The system (23) describes a vector field of the critical normal vector. An algorithm is constructed on the basis of the sets (16) and (23), that tests if any point on the contact lines is an ordinary node or singular point of second order [23, 29].

\subsubsection{Computer program, based on mesh region}

The last stage of the computer tooth surfaces synthesis aims at finding out the contact lines in the whole region of mesh. Elimination of the singular points of first order (ordinary nodes) in the pitch point vicinity was accomplished, by using one of the above considered criterion, concerning "critical angle". In this stage, we examine the whole region of mesh. The aim is to find out singular points of first and second order, or to establish that there are none.

A singular point of second order is characterized by an annular relative velocity vector, when the normal vector to the tooth surface in it does not exist. These points cause an undercutting of the Spiroid gear surface.

When changing the parameter of meshing (i.e. the angle of Spiroid pinion rotation) in a discrete way, the contact lines in a function of the one of the cylindrical coordinates, which describe the active tooth surfaces of the Spiroid pinion (Spiroid hob) are found out. This independent cylindrical coordinate describes an interval with a length of $2 L_{1} / m$ ( $L_{1}$ face width of pinion; axial module of teeth). For each of its values, the program verifies if the point's coordinates belong to the region of mesh or not. Analytical relations for existence of ordinary nodes or points of undercutting are verified, too.

Input data are the geometric and the technological parameters obtained in the optimization process of the designed gear pair. They are: number of Spiroid pinion threads, number of Spiroid gear teeth, offset, parameter of meshing, pinion reference circle radius, distance between the Spiroid pinion reference and the offset line of the gears, angle between axes, Spiroid pinion face width, tooth module. For each value of the parameter of meshing, which vary in a 
given interval, are written the number of the contact lines, the number of points on them, and a text according to the fact, whether there are or not ordinary nodes or points of undercutting among them. Computer program is organized following the scheme below (see Fig. 11).

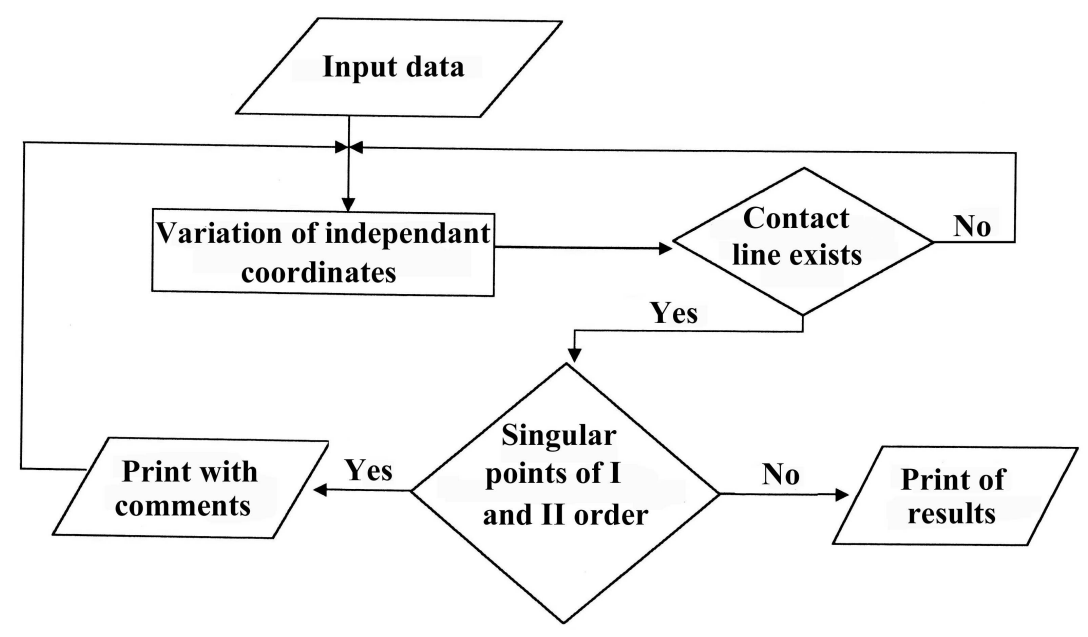

Fig. 11. Computer program for a synthesis, based on a region of mesh

\section{Conclusion}

The present paper gives a brief survey on the approaches, used to design mathematical models of spatial transmission synthesis. These approaches have been developed in the Institute of Mechanics of the Bulgarian Academy of Sciences and have been applied to model different types of gear drives with arbitrarily skewed axes. The application of the two models of spatial gear synthesis, the pitch contact point model and the mesh region model in Spiroid and Helicon gears is also illustrated. Algorithms and computer programs for the design of Spiroid and Helicon gear drives are elaborated. Synthesis, design, technological back-up and manufacture of various types of Helicon reducers are performed.

\section{REFERENCES}

[1] Bohle, F. Spiroid Gears. A New Development in Gears of the Skew-Axis Type. Machinery, 62 (1955), No. 2, 155-161. 
[2] SAARI, O. The Mathematical Background of Spiroid Gears. Industrial Mathematical Series, Detroit, 7 (1956), 131-144.

[3] Nelson, W. Spiroid Gearing. Part 1 - Basic Design Practices. Machine Design, 33 (1961), No. 4, 136-144.

[4] Nelson, W. Spiroid Gearing. Part 2 - Durability, Strength and Efficiency. Machine Design, 33 (1961), No. 5, 93-100.

[5] Nelson, W. Spiroid Gearing. Part 3 - Materials, Mounting Detail, Lubrication. Machine Design, 33 (1961), No. 6, 165-171.

[6] Dudley, D. Gear Handbook. The Design, Manufacture and Application of Gears, New York, McGraw-Hill, 1962, 1040.

[7] Razdevich, S. Dudley's Handbook of Practical Gear Design and Manufacture, Second Edition, CRC Pres Taylor\&Francis Group, 2011, 862.

[8] Georgiev, A. V. Elements of Geometric Theory and Some Questions of Design and Manufacture of Hypoid-Worm Gears, Ph. D Thesis, Izhevsk, 1965, 160 (in Russian).

[9] Georgiev, A., V. Goldfarb. S.Aspects of Geometric Theory and Investigation of Sprioid Gears with Cylindrical Pinion, Mechanika Machine, Moscow, Nauka, 1972, 70-80 (in Russian).

[10] Goldfarb, V. Aspects of Gears and Reductions Drives Automated Design, Gearing and Transmissions, Assoc. Engineers, Izhevsk, 1 (1991), 20-24 (in Russian).

[11] Schwagerl, D. Investigation of Helicon and Spiroid Gears with Trapezoidal Helical Profile by the Theory of Hertz and by Hydrodynamic Theory, Ph. D. Thesis, Mechanical and Electrical Faculty to Technical University of Munchen, 1967, 25 (in German).

[12] Bolos, V. Sprioid Worm Gearings. The Hobbing of Plane Vehicles, Targu Mures, Editura Universitatii "Petru Maior", 1999, 264 (in Romanian).

[13] Su Song, D., C. Gentle. Numerical Analysis of Sprioid Gears with Localized Tooth Contact Manufactured by a New Economical Method. International Journal of Gearing and Transmission, Special Issue, December 2000, UK, Nottingham, $2000,13-21$.

[14] Abadjiev, V. On the Synthesis and Analysis of Spiroid Gears, Ph. D. Thesis, Sofia, 1984, 158 ( in Bulgarian).

[15] Abadjiev, V. Aspects on Geometry Synthesis of High Reduction Helicon Gears. Strojnicky Casopis, Bratislava, 39 (1988), No. 2, 231-241.

[16] Abadjiev, V., D. Petrova. Synthesis Of Geometric Primary Pitch Circles of Externally Meshed, Skew-Axes Gears, Proc. of 15th Design Automation Conference of ASME, September 17-21, 1989 Volum Book No. H0509C, ASME, Canada, Montreal, Quebec, Design Engineering Division (Publication) DE, v 19-3, n pt 3, Advanced in Design Automation - 1989, Mechanical Systems Analysis, Design and Simulation, 1989, 241-245.

[17] Abadjiev, V. Aspects of Mathematical Modelling if Skew-Axes Gears whose Tooth Surfaces Have a Linear Contact. Gearing and Transmissions, Izhevsk Moscow, 2 (1994), 24-31. 
[18] Abadjiev, V. On the Kinematical Theory of the Spatial Transformation of Rotations. Engineering Mechanics, Brno, 2 (1995), No. 4, 255-259.

[19] Abadjiev, V., D. Petrova. Testing of Kinematic Conjugation of the Flanks of Gear-Pairs of Type Spiroid. Journal of Mechanics and Machine Theory, Pergamon, 32 (1997), No. 3, 343-349.

[20] Abadjiev, V. Mathematical Modelling for Synthesis of Spatial Gears. Journal of Process Mechanical Engineering, Proc. Inst. Mech. Engrs, 216 (2002), Part E, $31-46$.

[21] Abadjiev, V., D. Petrova. Analytical and Computer Design of Spiroid Gears, M. Heilio (ed.), Proc. of 5th European Conference on Mathematics in Industry, Printed in Netherlands, B. G. Teubner Stuttgard and Kluwer Academic Publishers, 1991, 121-126.

[22] Abadjiev, V., D. Petrova. On the Synthesis and Computer Design of Spiroid Gears: A Review of the Bulgarian Approach. Journal of Theoretical and Applied Mechanics, 33 (2003), No. 3, 3-30.

[23] Abadjiev, V. Gearing Theory and Technical Applications of Hyperboloid Mechanisms, Sc. D. Thesis, Sofia, Institute of Mechanics, Bulgarian Academy of Sciences, 2007, 309 (in Bulgarian).

[24] Abadjiev, V., E. Abadjieva, D. Petrova. Non-Orthogonal Hyperboloid Gears. Synthesis and Visualization of Pitch Configurations with Normal Orientation. Compt. rend. Acad. bulg. Sci., Sofia, 64 (2011), No. 9, 1311-1319.

[25] Abadjiev, V., E. Abadjieva, D. Petrova. Pitch Configurations: Definitions, Analytical and Computer Synthesis. ASME, (2011), Paper No. DETC2011-47562.

[26] Abadjieva, E. Spatial Rack Drives. Mathematical Modelling for Synthesis, VDM Verlag Dr. Müller e.K., 2011, 72.

[27] Litvin, F. Theory of Gearing, Moskow, Nauka, 1968, 584 (in Russian).

[28] Litvin, F. Gearing Geometry and Applied Theory. New Jarsy 07632, Englewood Eliffs, PTR Prentice Hall, A Paramount Communication Company, 1994, 724.

[29] Abadjiev, V., D. Petrova. Testing of Kinematic Conjugation of the Flanks Active Surfaces of Gear-Pairs of Type Spiroid. Journal Mechanism and Machine Theory, 32 (1997), No. 3, 343-349. 